\title{
A Three Dimensional Representation of the Fracture Network of a CO2 Reservoir Analogue (Latera Caldera, Central Italy)
}

\author{
A. Alemanni*, M. Battaglia*, S. Bigi*, E. Borisova ${ }^{\circ}$, A. Campana ${ }^{\circ}$, M. Loizzo $^{\circ}$ \& S. \\ Lombardi
}

*Dipartimento di Scienze della Terra, Università "La Sapienza" P.le Aldo Moro, 5, 00185, Rome, Italy.

${ }^{\circ}$ Etudes et Productions Schlumberger, Clamart, France

\begin{abstract}
Fault zones as well as fractures, whether developed in sediments, sedimentary or crystalline rocks, are structurally heterogeneous. Building models of fractures media it is not a simple task. The high degree of heterogeneity occurring in fractured reservoirs can make difficult to predict the distribution of porosity and permeability in the system. An approach based only on statistical and numerical methods may include a high grade of uncertainty (e.g., Billaux et al., 1989). Data from outcrops representative of reservoir rocks can help reducing uncertainties and increasing confidence in numerical and statistical models.

The purpose of this work is to create simulation properties with the power to predict the reservoir behaviour in term of permeability and fluid flow simulation. By modeling the fractures explicitly, one can honour the spatial relationships between properties in adjacent cells. Fracture modeling is a multi-step process involving several disciplines within reservoir characterization and simulation.
\end{abstract}

(C) 2011 Published by Elsevier Ltd. Open access under CC BY-NC-ND license.

Keywords: Type your keywords here, separated by semicolons ;

\section{Introduction}

Fault zones as well as fractures, whether developed in sediments, sedimentary or crystalline rocks, are structurally heterogeneous. Building models of fractures media it is not a simple task. The high degree of heterogeneity occurring in fractured reservoirs can make difficult to predict the distribution of porosity and permeability in the system. An approach based only on statistical and numerical methods may include a high grade of uncertainty (e.g., Billaux et al., 1989). Data from outcrops representative of reservoir rocks can help reducing uncertainties and increasing confience in numerical and statistical models.

The purpose of this work is to create simulation properties with the power to predict the reservoir behaviour in term of permeability and fluid flow simulation. By modeling the fractures explicitly, one can honour the spatial 
relationships between properties in adjacent cells. Fracture modeling is a multi-step process involving several disciplines within reservoir characterization and simulation.

\section{The caldera of Latera case study}

The Caldera of Latera, located close to the Tyrrhenian coast of Italy, is characterized by a high geothermal gradient and an extensional volcano-tectonic regime. These settings explain the continue production of $\mathrm{CO} 2$, probably due to thermo-metamorphic reactions at depth, and the subsequent migration of $\mathrm{CO} 2$ to the surface along faults and fracture systems. Previous studies carried out in this area were able to describe the migration to surface of deep, naturally produced $\mathrm{CO} 2$ along various buried and exposed faults in the Latera caldera (Central Italy) by integrating structural geology and near-surface gas geochemistry surveys (Annunziatellis et al., 2008). Results showed that gas migration is channeled along discrete, high-permeability pathways within the faults, with the release occurring from spatially-restricted gas vents. It is worth noting that gas migration can change drastically along strike.

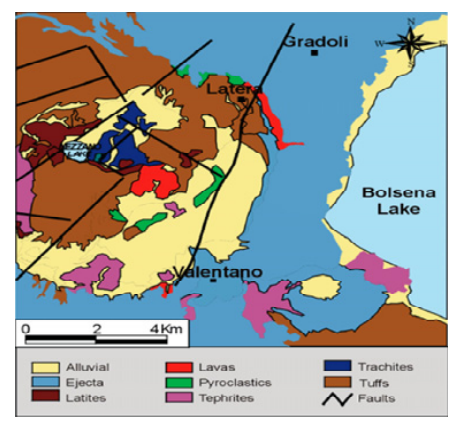

Figura 1 - Geological map of Latera Caldera

\section{2D mapping of fractures}

Spatial data were collected using several sampling windows of appropriate dimension (approximately $0.5 \mathrm{~m}$ ) on adjacent rock faces of different, and where possible, orthogonal orientation. The interpreted individual fractures were combined and represented by lines (Figs. 2 and 3). In this way, they were easily imported and manipulated into a CAD.
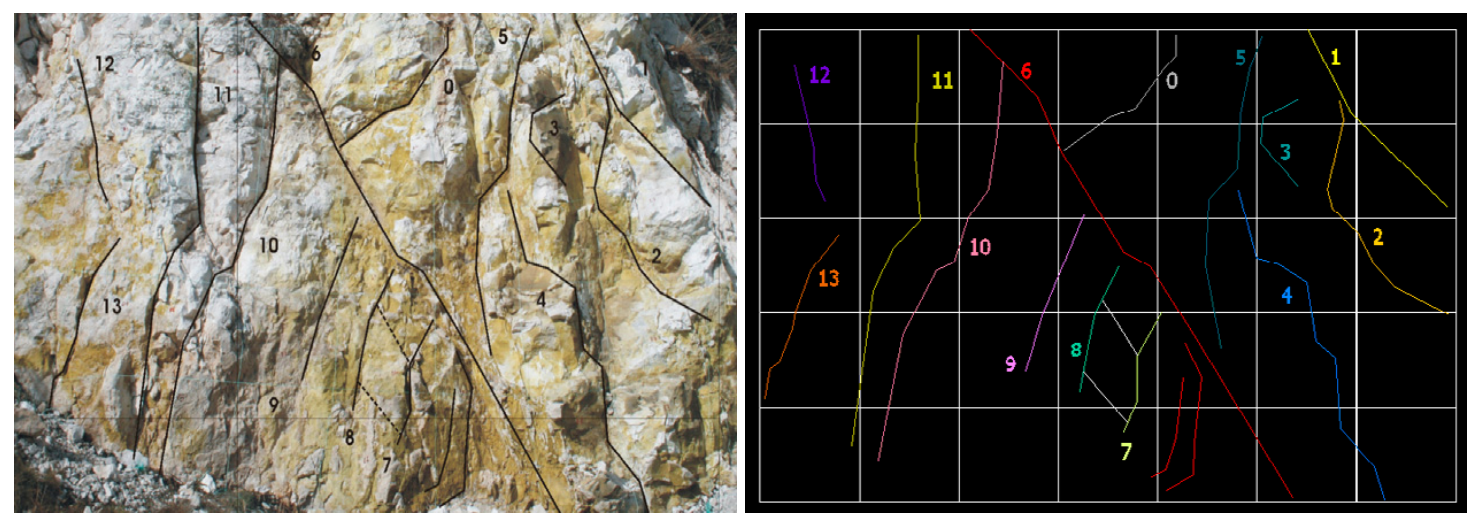

Figura 2 - Two-dimensional mapping of the first wall of the outcrop. The sampled area is approximately 3.5 by 2.5 meters. 

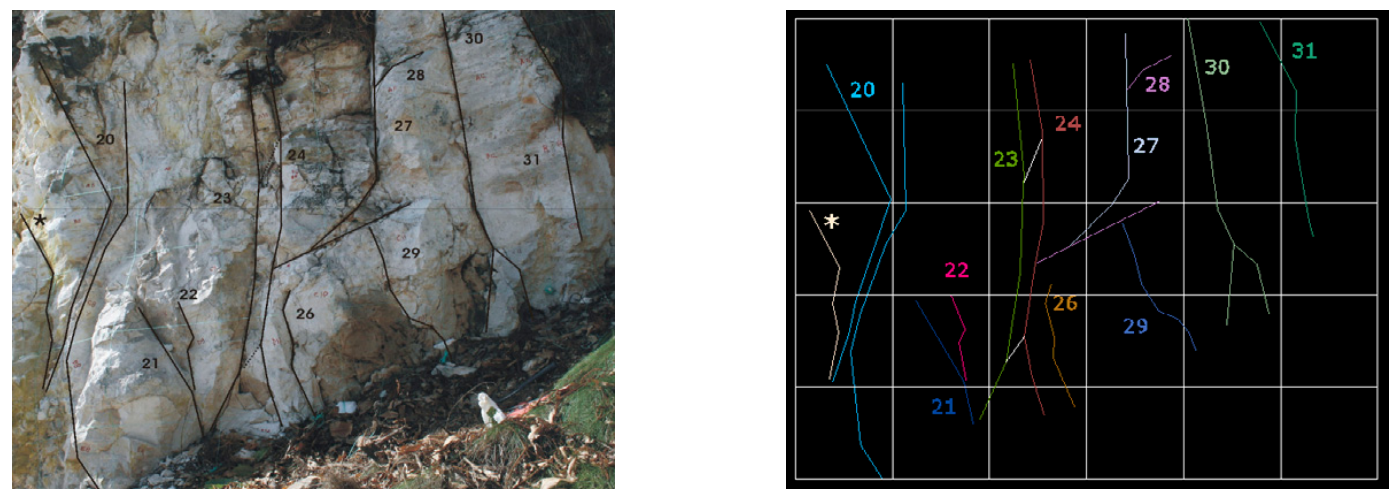

Figura 3 - Two-dimensional mapping of the second (orthogonal) wall of the outcrop. The sampled area is approximately 3 by 2.5 meters

\section{3D reconstruction of fractures network}

The interpreted fractures from a $3.5 \times 3 \times 2.5 \mathrm{~m}$ outcrop (Fig. 4a) were imported into a CAD to create a three dimensional fracture network. The reconstruction of the fractured volume was completed applying a fractures classification based on their structural and genetic features. This classification had also helped to establish a correlation between the structural features and the permeability of the measured fractures and fault planes. To produce the 3D model, the fractures were correlated between profiles, interpolated as continuous surfaces and then plotted in 3D (Fig. 4). The advantage of such a representation is the ability to image the fractures as warped surfaces. The picking and correlation of lines identifying each fracture was done manually, and therefore, constituted the most time-consuming part of this work. The surface corresponding to a fracture was calculated from the correlated picked lines by using an inverse distance interpolation on a regular-spaced grid. The grid spacing is $0.5 \times 0.5 \mathrm{~m}$. Finally, all surfaces were represented with different colours to provide a better visual impression.
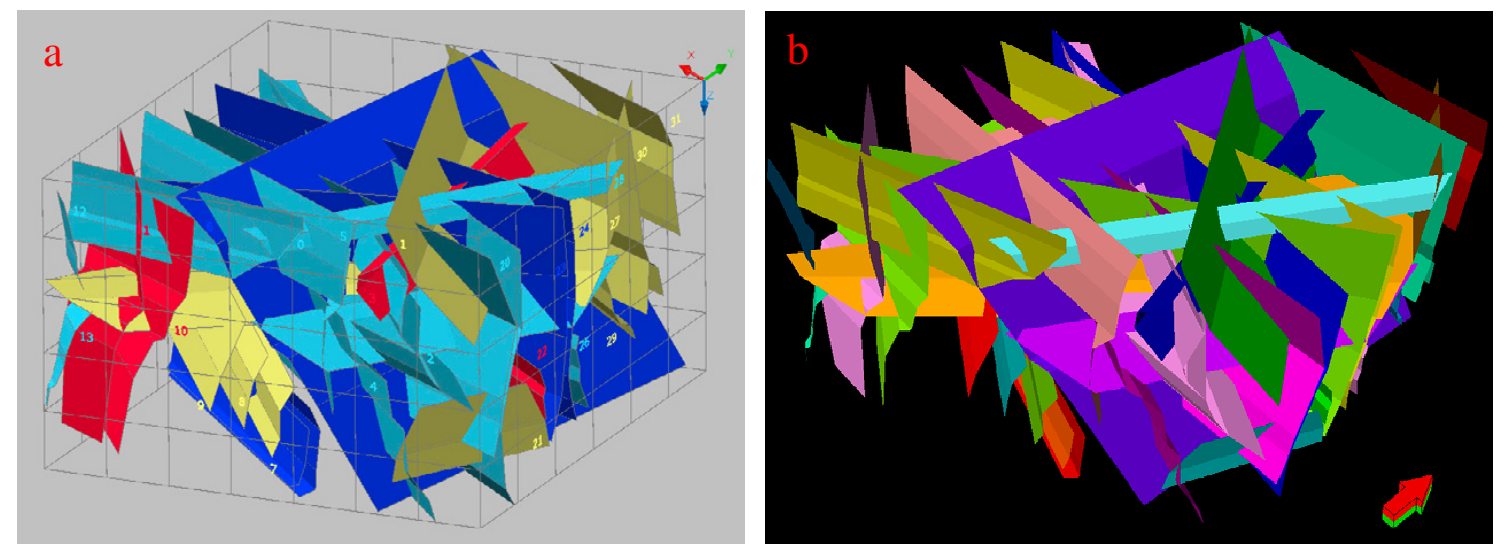

Figura 4 - 3D representation of the fracture network of the outcrop, created with AutoCAD (a) and with Petrel (b). 


\section{Deterministic and stochastic models construction and comparison. Main preliminary results}

\subsection{Deterministic model}

The simplest fracture sets are defined deterministically as a group of previously defined fractures, either as a result of fault plane interpretation extraction from a seismic cube, a previously defined discrete fracture network in Petrel, fractures imported (as in this work), and use them directly in the discrete fracture network.

If a deterministic method is used, it needs to know where and how the fractures behave in the 3D space, and could be done using high confidence fracture patches from an existing fracture model. By modeling the fractures explicitly, it can honour the spatial relationships between properties in adjacent cells (Fig. 5). When this method is used, Petrel will perform an approximation of the input fracture plane when it deviates from being planar.

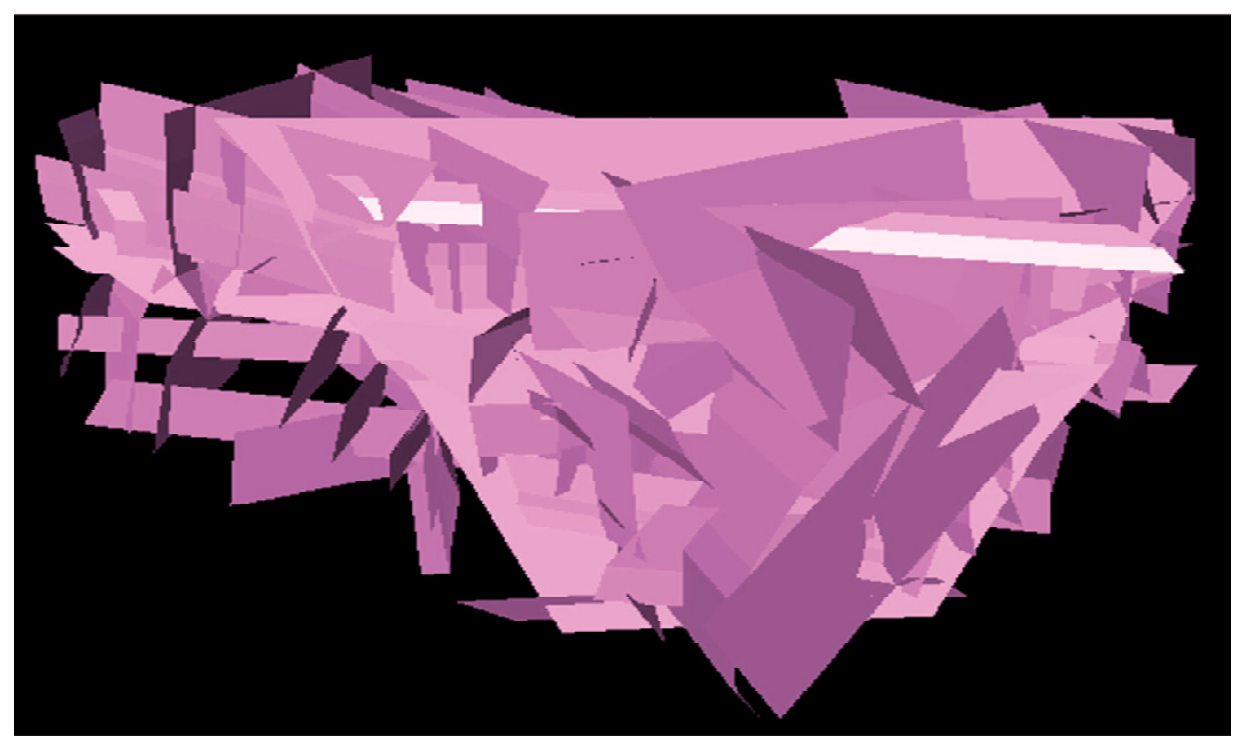

Figura 5 - Deterministic model reconstructed in Petrel

\subsection{Stochastic Model}

Fractures modeled stochastically can be described statistically using either numerical input, surfaces, or grid properties. Properties in the 3D grid can vary and can easily be modeled using standard algorithms. Because the method is stochastic, it requires a seed point from a random number generator. If the Random seed is fixed, Petrel will regenerate the same results. If not, Petrel will create a new equally probable output that honors all the input parameter settings. Stochastic method are based on random theory so there is more than one realization as a result. Using numerical inputs is quicker and is a good way of testing the outcomes and building scenarios. Grid properties can vary in $3 \mathrm{D}$ and it is possible to have many options in terms of generating these properties based on a range of input data. Some inputs need to define before Petrel can create some sensible fracture outputs, as distribution, geometry and orientation of fractures. The fracture distribution (fracture intensity) is defined as the amount of fracturing per unit volume. This can be expressed as the number of fractures per volume, fracture length per volume, or fracture area per volume (P 32). For a given fracture distribution, the constant which relates spacing to P32 will not change. In each cell of the model, the P32 value gives the expected area of fractures within the cell divided by the volume of the cell. The fracture geometry (the Shape control) in Petrel allows for a simplification of the fault 
plane. The fracture orientation uses mean dip and azimuth and concentration as input to the Fisher model (the angular equivalent of a normal distribution when the concentration equates to a standard deviation).

\subsection{Simulation of properties}

The purpose of modeling fractures is to create simulation properties with the power to predict the reservoir behaviour.

Modeling flow in fractured reservoirs is difficult. The challenge requires a software solution that supports tight integration between the static and dynamic reservoir modeling disciplines and provides a way to visualize and analyze many data types that may be direct or indirect indicators of fractures.

The ultimate goal is to find 3D grid properties which describe permeability and porosity for fracture, as well as the standard permeability and porosity are gotten from the matrix. With a DFN we also get an idea about the permeability anisotropy in the model.

Once data have been identified, analyzed and categorized, the fracture model itself can be built.

It was assigned at each fracture an aperture value and consequently, basing on the Darcy's law, it was estimated the permeability values:

\section{Permeability $=(\text { Aperture })^{2} / 12$}

The permeability was calculated according to the geometry and distribution of fractures in each cell.

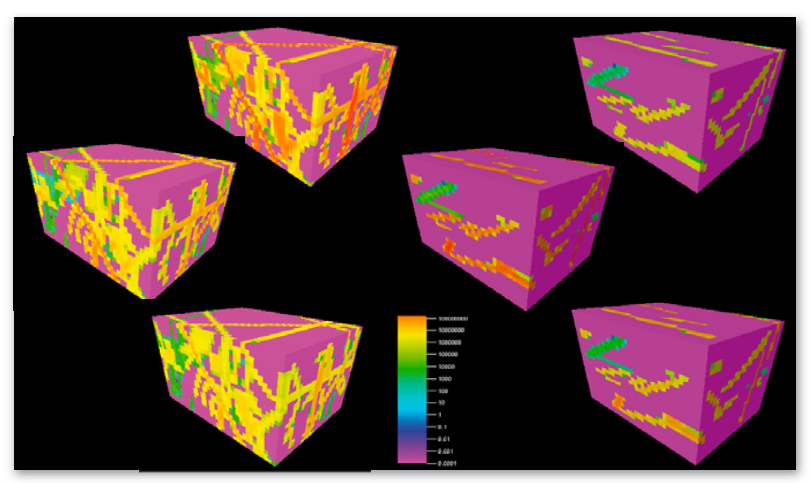

Figura 6 - Permeability properties distribution obtained with Petrel in the different three direction (x, y and z)

\subsection{Main results}

The graphic of Fig. 7 shows the different values of permeability for the deterministic and the stochastic model. The stochastic model of permeabilities distribution, calculated along each three directions are underestimated by comparison with deterministic model permeabilities distribution described in the previous paragraph. These differences are due to the lower number of fractures generated by Petrel using in the stochastic methods compared to the real number of fractures provided by the deterministic model.

Permeability values for each models are higher along the y-direction, corresponding to the intersection of the main fractures sets measured in the deterministic model. That means that the principal migration path of gas occurs rather horizontally along the intersection of the principal fracture planes. 


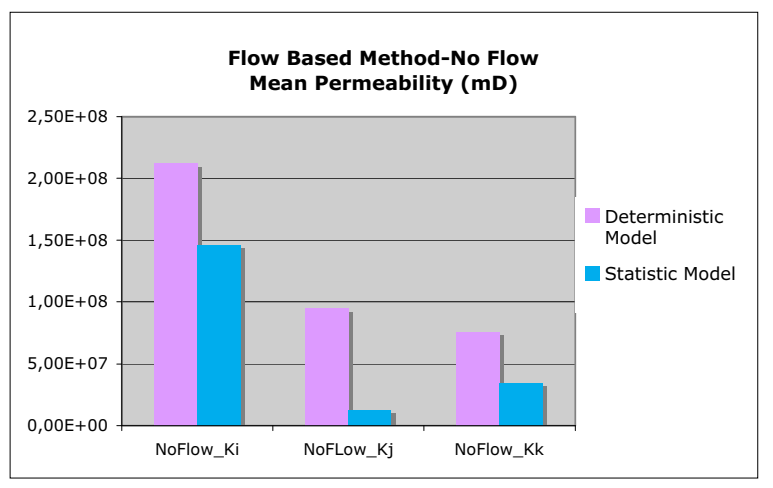

Figura 7 - Comparison between the obtained values of permeability distribution calculated for deterministic and statistic model.

\section{References}

Billaux, D., J. P. Chilès, K. Hestir and J. Long.. Three-Dimensional Statistical Modelling of a Fractured Rock Mass - An Example From the Fanay-Augères Mine,” Int. J. Rock Mech. \& Min. Sci., 1989, 26(3-4), 281-299.

Annunziatellis A., Beaubien S.E., Bigi S., Ciotoli G., Coltella M. and Lombardi S. Gas migration along fault systems and through the vadose zone in the Latera caldera (central Italy): implications for CO2 geological storage. Int. J. Of Greenhouse gas control, 2008,

doi:10.1016/j.ijggc.2008.02.003. 